\title{
Special features of the 2009 pandemic swine-origin influenza A H1N1 hemagglutinin and neuraminidase
}

\author{
VAVRICKA Christopher John ${ }^{1 *}$, LIU Yue ${ }^{1}$, LI Qing ${ }^{1,2}$, SHI Yi $^{1,3}$, WU Yan ${ }^{1}$, SUN YePing ${ }^{1}$, \\ QI JianXun ${ }^{1} \&$ GAO George $\mathrm{Fu}^{1,2,3,4^{*}}$ \\ ${ }^{1}$ CAS Key Laboratory of Pathogenic Microbiology and Immunology, Institute of Microbiology, Chinese Academy of Sciences, Beijing 100101, \\ China; \\ ${ }^{2}$ School of Life Sciences, University of Science and Technology of China, Hefei 230027, China; \\ ${ }^{3}$ Graduate University of Chinese Academy of Sciences, Beijing 100049, China; \\ ${ }^{4}$ Beijing Institutes of Life Sciences, Chinese Academy of Sciences, Beijing 100101, China
}

Received March 14, 2011; accepted April 11, 2011; published online May 10, 2011

\begin{abstract}
Since the 2009 pandemic H1N1 swine-origin influenza A virus (09 S-OIV) has reminded the world about the global threat of the ever changing influenza virus, many questions regarding the detailed re-assortment of influenza viruses yet remain unanswered. Influenza A virus is the causative agent of the pandemic flu and contains 2 major antigenic glycoproteins on its surface: (i) hemagglutinin (HA); and (ii) neuraminidase (NA). The structures of the 09 S-OIV HA and NA proteins (09H1 and 09N1) have recently been resolved in our laboratory and provide some clues as to why the 09 S-OIV re-assortment virus is highly infectious with severe consequences in humans. For example, the 09H1 is highly similar to the HA of the 1918 influenza A pandemic virus in overall structure and especially in regards to its 5 defined antibody binding epitopes. For 09N1, its most distinctive feature is the lack of a 150-loop active site cavity, which was previously predicted to be present in all N1 NAs, and we hypothesize that the 150-loop may play a important role in the substrate specificity ( $\alpha 2,3$ or $\alpha 2,6$ linked sialic acid receptors) and enzymatic mechanism of influenza NA. Combination of the HA and NA with special characteristics for the 09 S-OIV might contribute to its high increased transmissibility in humans.
\end{abstract}

2009 pandemic H1N1, hemagglutinin (HA), neuraminidase (NA), swine-origin influenza virus (S-OIV), reassortment

Citation: Vavricka C J, Liu Y, Li Q, et al. Special features of the 2009 pandemic swine-origin influenza A H1N1 hemagglutinin and neuraminidase. Chinese Sci Bull, 2011, 56: 1747-1752, doi: 10.1007/s11434-011-4517-9

In early 2009, severe cases of H1N1 influenza A virus broke out primarily in Mexico. On April 29, 2009, the World Health Organization (WHO) raised its alert level for this influenza outbreak to Phase 5, the highest level ever announced since the warning system was introduced in 2005 (WHO). By June 11, 2009, this pandemic H1N1 virus had officially been reported in 74 countries and the WHO alert level was then raised to Phase 6, the pandemic level (WHO). In 2010, reports of pandemic H1N1 infection significantly declined and on August 10, 2010 the WHO declared that the pandemic was over (WHO). As of August 6,

*Corresponding authors (email: chris@im.ac.cn; gaof@im.ac.cn)
2010, the 2009 pandemic H1N1 had been attributed to the deaths of over 18449 individuals (WHO).

Previous influenza pandemics include the 1918 'Spanish Flu' (H1N1), 'Asian Flu' of 1957 (H2N2), and 'Hong Kong Flu' of 1968 (H3N2) [1]. Like the 2009 pandemic virus, the 1918 virus was also an H1N1 influenza A virus and it is estimated to have led to the deaths of over 40 million people (WHO). The 1957 H2N2 Asian flu is estimated to have killed about 2 million people and the 1968 H3N2 Hong Kong flu is estimated to have killed about 1 million people, (WHO). Although the $2009 \mathrm{H} 1 \mathrm{~N} 1$ virus was raised to the pandemic level by the WHO, only about 20000 deaths have been attributed to it, comparable to that of the seasonal flu 
(WHO). However, like the 1918 pandemic virus, the 2009 pandemic virus often leads to severe respiratory problems in young, pregnant and healthy individuals [2].

Influenza viruses can be categorized into 3 types: A, B and $\mathrm{C}$. Influenza A virus is the causative agent of pandemic flu. The influenza A virus genome contains 8 RNA segments which encode up to 12 proteins: NP, NS1, NS2, M1, PA, PB1, PB2, PB1-F2, PB1 N40 and 3 surface proteins, $\mathrm{M} 2$, HA and NA [1,3]. HA (hemagglutinin) and NA (neuraminidase) are the principal influenza surface antigens and contain multiple serotypes based upon their antigenicity [1]. HA is responsible for sialic acid receptor binding, facilitating the process of virus and host endosomal membrane fusion and allowing the viral genetic materials to gain entry into the cytoplasm [1]. NA, which is a sialidase, catalyzes hydrolysis of terminally linked sialic acid receptors and functions as the receptor destroying element of influenza A and B viruses, thereby helping newly formed virions to break free from infected cells and to continue infecting new cells [1]. HA is grouped into 16 different known serotypes and NA is grouped into $9[1,4]$. Therefore influenza A virus strains are named based upon their combination of HA and NA (e.g. H1N1, H5N1, H3N2) [1]. Certain HA/NA virus types are highly specific for different animal hosts which is promoted, partially, by evolutionary differences in host immune systems and host restriction factors [4,5]. For example, H5N1 is highly common in birds whereas H3N2 is a common seasonal flu strain in humans [5]. Furthermore, human seasonal flu strains produce more variation in their $\mathrm{HA} / \mathrm{NA}$ antigenic sites due to a higher selective pressure in the human immune system [6].

During infection of a host with multiple influenza A virus strains, reassortment of RNA segments from different viruses into new budding virions may occur [7]. The 2009 pandemic swine-origin H1N1 influenza A virus (09 S-OIV) is a reassortment with $\mathrm{PB} 2$ and PA derived from avian influenza, PB1 derived from human $\mathrm{H} 3 \mathrm{~N} 2, \mathrm{HA}, \mathrm{NP}$ and NS derived from classical swine $\mathrm{H} 1 \mathrm{~N} 1$, and NA and $\mathrm{M}$ derived from a Eurasian avian like swine H1N1 influenza lineage [8-10]. Both the $1957 \mathrm{H} 2 \mathrm{~N} 2$ pandemic and the $1968 \mathrm{H} 3 \mathrm{~N} 2$ pandemic were also reassortments between avian and human viruses [11]. Highly pathogenic avian influenza viruses, such as $\mathrm{H} 5 \mathrm{~N} 1$ are often very deadly to humans, however infection of human with these avian influenza viruses is rare [12] Recently it has been confirmed that multiple reassortments of avian $\mathrm{H} 5 \mathrm{~N} 1$ with human $\mathrm{H} 3 \mathrm{~N} 2$ can lead increased virulence in mice [13]. It is therefore a serious concern that highly pathogenic avian influenza strains may gain higher transmissibility in humans through an intermediate mixing vessel; especially swine [14].

According to the current understanding of influenza virus interspecies transmission, swine may act as a primary mixing vessel between avian and human virus strains [14]. HA and NA receptor specificities likely play an integral role in determining the basis for influenza host specificity. In gen- eral, avian hosts primarily contain $\alpha 2,3$ linked sialic acid receptors and avian influenza virus preferentially binds the $\alpha 2,3$ receptor, however the upper respiratory tract of humans contains primarily $\alpha 2,6$ linked sialic acid receptors [15-17]. This may explain why human influenza viruses preferentially bind the $\alpha 2,6$ receptor and is considered a major reason why avian influenza virus is not highly transmissible in humans. Swine contain both $\alpha 2,3$ and $\alpha 2,6$ linked sialic acid receptors in their respiratory system and possess a less complex immune system than humans, which may explain why they are more susceptible to infection by avian and human strains of influenza and why swine influenza viruses may easily evolve the ability to bind the human $\alpha 2,6$ receptor [15-17]. The recent 2009 pandemic H1N1 is the first pandemic influenza re-assortment virus with a primarily swine-influenza origin, which is a major reason why it has caused great concern [18-21].

Although other influenza proteins like PB1 and PB2 also play an important role in pathogenicity, HA and NA are on the front line of the process of virus entry, fusion and release of progeny virions. Furthermore, these two viral envelope glycoproteins are the primary targets of the immune system and interact directly with the receptors, and therefore are critical elements in determining host cell specificity $[1,4]$. For these reasons, the unique properties of the $09 \mathrm{H} 1$ and $09 \mathrm{~N} 1$ can provide many insights into the increased transmissibility and pathogenicity of the 09 S-OIV.

\section{HA}

HA binds primarily to $\alpha 2,3$ or $\alpha 2,6$ linked sialic acid receptors on the mammalian cell surface acting as the receptor binding element of type A and B influenza viruses $[1,4]$. The receptor binding function of HA is the first step of the influenza infection cycle and there has been a great deal of research into its function [22]. The sialic acid binding site is located in the head region of each HA molecule [22]. Influenza HA contains various $\mathrm{N}$-glycosylation sites and is secreted as a homotrimer to the membrane/viral envelope where it is anchored via transmembrane helices [22,23]. Cleavage of each HA monomer into two polypeptides, HA1 and HA2, by cellular proteases is necessary for HA to facilitate host-virus membrane fusion [22].

Sequence analysis indicates that $09 \mathrm{H} 1$ has recently evolved and that two major subtypes of 09 S-OIV emerged, with a Mexico and New York origin [24,25]. The structure of 09HA has been reported by several research groups and the detailed structure is strikingly similar to 1918 pandemic H1 HA (18H1) [6,26,27]. The similarity is especially pronounced in regards to the 5 defined antibody binding sites $[6,7]$. This can explain why older generations which have been exposed to the 1918 pandemic H1N1 have resistance to $09 \mathrm{~S}-\mathrm{OIV}$ [26]. In a similar manner, it has recently been 
reported that survivors of the 09 S-OIV have acquired an effective immune response against many influenza strains [28]. Furthermore, neither 18H1 nor 09H1 contain conserved $N$-glycosylation sites in the head region which may interfere with receptor binding and the lack of $\mathrm{N}$-glcosylation in the head region has been shown to increase their receptor binding affinities [29].

Another primary similarity of $18 \mathrm{H} 1$ with $09 \mathrm{H} 1$ is the sialic acid receptor specificity, which is the critical determinant of host range as demonstrated in numerous previous studies [30,31]. Most importantly, the 190 and 225 residues (all HA residues here are $\mathrm{H} 1$ numbered) have been identified to play a predominant role in determining binding specificity of H1 [32]. For 09H1, the Asp190/Asp225 pair is responsible for specifically recognizing only the $\alpha 2,6 \mathrm{hu}-$ man receptor (unpublished data), whereas other matching patterns such as Glu190/Gly225 and Asp190/Gly225 are regarded as crucial factors for binding $\alpha 2,3$ receptors in avian hosts and both receptors in swine, respectively [22,33,34]. Moreover, recent analysis using glycan microarrays also has revealed that Asp225Glu/Gly substitution results in slightly or moderately enhanced binding affinity towards a broader range of different $\alpha 2,3$ receptor analogs [35]. Based on the binding modes in $30 \mathrm{H} 1$ and $34 \mathrm{H} 1$ with the human and avian receptors [32], 09H1 with the Gly225 substitution may bind to both human and avian receptors [36]. Regarding the Asp225Glu substitution, it was hypothesized that the longer glutamate side chain might facilitate the interactions with Gal-2 of the $\alpha 2,3$ avian receptor [36], however, in our recent unpublished studies, the Asp225Glu mutant of $09 \mathrm{H} 1$ preferentially binds to the $\alpha 2,6$ human receptor due to a different mechanism.

09H1 contains additional special features worth mentioning that distinguish it from other HA proteins, including 18H1. For example, 09H1 contains larger basic patches relative to all other reported HA structures (Figure 1) [6]. The His/Lys basic patch is also relatively strong in the $18 \mathrm{H} 1$ structure and is hypothesized to be involved in facilitating membrane fusion [36,37]. Additionally, there is an extra $\mathrm{N}$-glycosylation site in $09 \mathrm{H} 1$ close to this very basic patch (Figure 1) [6]. This $N$-glycosylation site may modulate the effect of the stronger basic patch or may play a role in masking antibody recognition. The precise effects of the basic patch and additional $N$-glycosylation site on the function of HA deserve further research and investigation.

\section{NA}

There are currently 9 different serotypes of NA, which can be further classified into two separate groups based upon phylogenetic sequence analysis. Group 1 consists of $\mathrm{N} 1$, $\mathrm{N} 4, \mathrm{~N} 5$ and N8, and group 2 consists of N2, N3, N6, N7 and N9 [38]. Before the structure of 09N1 was released, all published group 1 neuraminidase structures contained an addi-

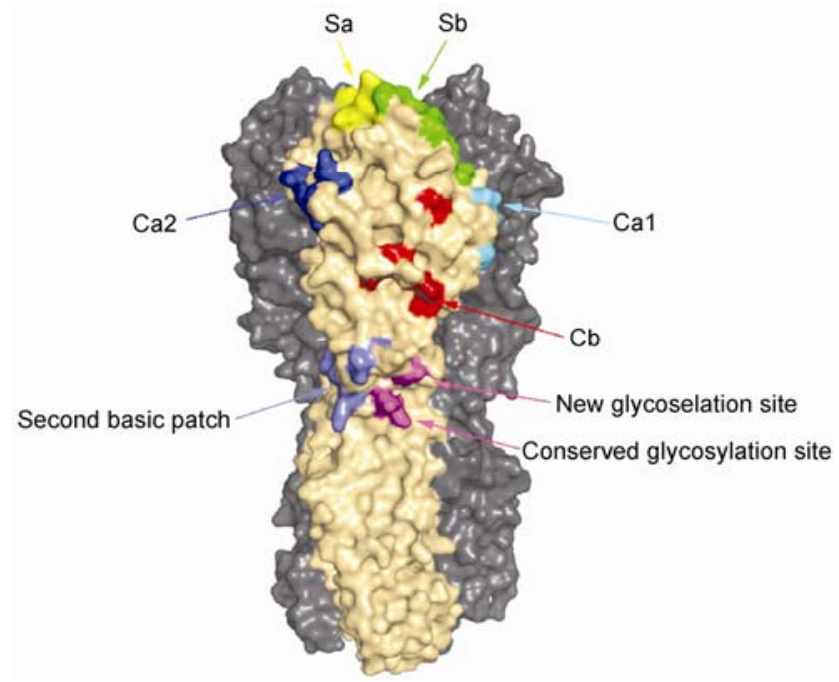

Figure 1 Overall structure of $09 \mathrm{H} 1$ with an emphasis on the classical antigenic sites, second basic patch and $N$-glycosylation sites adjacent to the second basic patch. Modified from Zhang et al. [6] (with permission from Protein \& Cell).

tional 150-loop cavity, which is not present in all other currently reported group 2 neuraminidase structures [38]. This led to the correlation of this structural feature with the primary sequence of NA [38]. However, our recent work has revealed that the $09 \mathrm{~N} 1$ crystal structure actually does not contain the 150-loop cavity (Figure 2) [39]. This indicates that the formation of the 150-cavity (at least in the crystal state) may possibly be influenced by a single amino acid at position 149 (Ile or Val, all NA numbering here is based on N2) and its coordination with the 430-loop, rather than entire NA amino acid sequence [39]. Nevertheless, in the case of N2 structures, a salt bridge between Asp147 and His150 appears to be the major factor in stabilizing the 150-loop in its closed conformation [39]. Still, the 150-loop is likely very flexible and should adopt various open and closed conformations in solution [40]. However, the 09N1 150-loop may be thought of as an intermediate between the typical group 1 NAs with the 150-loop cavity in their crystal structures and typical group 2 NAs with no 150-loop cavity.

There is a highly conserved network of electrostatic interactions between the NA active site and its ligand, sialic acid, consisting of residues Arg118, Glu119, Asp151, Arg152, Trp178, Arg224, Glu276, Glu277, Arg292, Arg371 and Tyr406. These amino acid residues are $100 \%$ conserved in all of the current available NA structures from both groups (although Arg292 is replaced by Lys in an artificial drug resistant Australia N9 strain) [41,42]. Most of these residues are involved in substrate binding or may stabilize the enzymatic transition state; however Asp151, which is in the 150-loop, and Tyr406 are likely to directly participate in the catalytic mechanism (based upon mechanistic studies in bacterial NA), although it is possible that water molecules may replace the function of these two residues leading to alternative mechanisms [41,43-45]. 
(a)

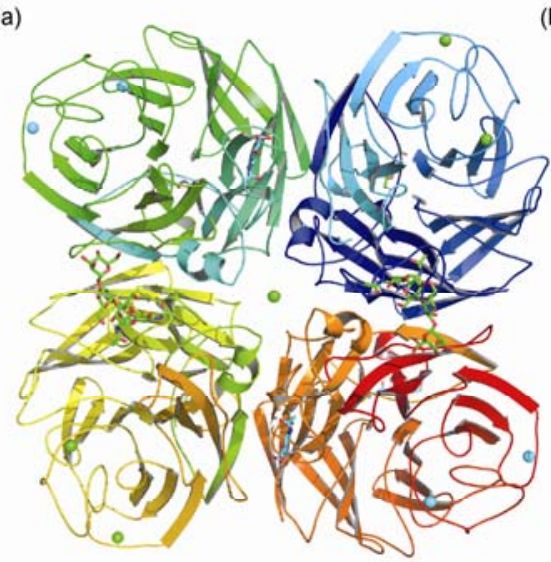

(b)
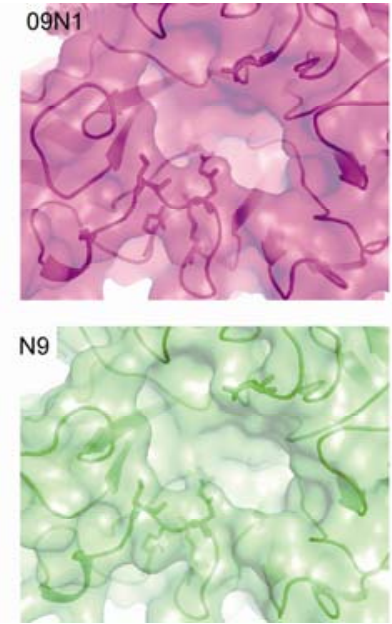
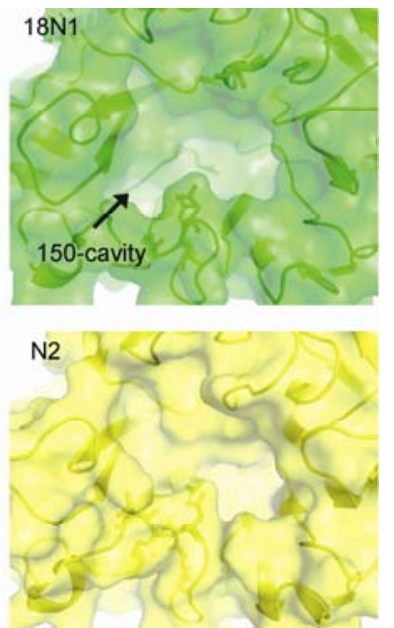

Figure 2 Overall structure of the 09N1 tetramer (a) and comparison of the active sites of 09N1, 18N1, N9 and N2, illustrating the 150-cavity deficient active site of $09 \mathrm{~N} 1$ (b).

We hypothesize that the 150-loop is important for substrate binding and that the conformation of the 150-loop plays an important role in determining the specificity of NA for $\alpha 2,3$ or $\alpha 2,6$ linked sialic acid. The ability to obtain the first sialic acid complex structure with a group 1 NA using 09N1 may indicate the importance of the 150-loop in substrate binding (unpublished data). The 150-loop of influenza NA contains a strictly conserved Asp151 residue, which we also hypothesize may be directly involved in the sialiside hydrolase mechanism of influenza NA. In the influenza NA-sialic acid complex structures, Asp151 participates in multiple hydrogen bonds with sialic acid, including the anomeric hydroxyl, which forms the glycosidic bond in the $\alpha 2,3$ or $\alpha 2,6$ receptor. Asp151 in the NA structures with an open 150-loop conformation is shifted over $1 \AA$ from the sialic acid binding site relative to the structures with a closed 150-loop conformation (Figure 3). Based on these observations, we propose a 3 step model: (i) the flexible 150-loop may assist binding of $\alpha 2,3$ or $\alpha 2,6$ linked substrate in the open conformation; (ii) hydrolysis of the glycosidic bond may be facilitated by movement of the loop back toward the substrate via Asp151; and (iii) release of product sialic acid via movement of the 150-loop into the open conformation. In this way the 150-loop may act as a switch between substrate binding, subsequent NA activity and release of product sialic acid. We speculate that 09N1 may be like an intermediate state between the typical group 1 (150-cavity) and group 2 (no 150-cavity) structural types and this special feature of $09 \mathrm{~N} 1$ may confer some advantage to the $2009 \mathrm{H} 1 \mathrm{~N} 1$ pandemic virus which is highly transmissible in humans. Clearly, more detailed biochemical and structural studies are needed to test these hypotheses.

\section{Discussion and perspective}

Because HA and NA bind sialic acid in an independent



Figure 3 Comparison of 150-loop orientations and the position of Asp151 using structures from all the currently available NA serotypes in the protein data bank (PDB) $(\mathrm{N} 4, \mathrm{~N} 8,18 \mathrm{~N} 1$ and avian N1 with an open 150-loop conformation; N2, N6, N9 and 09N1 with a closed 150-loop conformation). The distance between Asp151 in the open and closed conformations is over $1 \AA$. Sialic acid (yellow) is included from the Tokyo N2 structure (2BAT). Conserved Asp151 residues from structures with an open 150-loop conformation are colored green and Asp151 from structures with a closed 150-loop conformation are colored blue. PDB IDs: 18N1 3BEQ, avian N1 - 2HTY, Tokyo N2 - 2BAT, N4 - 2HTV, N6 - 1VOZ, N8 - 2HT5 and Australia N9 - 1NCA.

manner, it should not be possible that NA cleaves the sialic acid glycosidic bond while it is bound to HA. Therefore, there must be a critical balance between the expression levels, substrate binding, specificities and activities of each HA-NA pair. If the affinity of HA to its receptor is too high relative to the NA affinity toward the same receptor, the virus will be efficient at infecting new cells, but ineffective 
at release and vice versa. Although immunity is also an important factor, this may explain why certain HA-NA serotype combinations are more common than others and some are never observed. Recent data suggests that combination of $09 \mathrm{~N} 1$ with $18 \mathrm{H} 1$ results in a higher infectivity, however the 09H1 with 18N1 (1918 pandemic N1) does not (Figure 4) [46]. Because $09 \mathrm{H} 1$ and $18 \mathrm{H} 1$ are highly similar, it is difficult to say the advantage of $18 \mathrm{H} 1$ in this system. Perhaps the stronger basic patch and extra $N$-glycosylation site of $09 \mathrm{H} 1$ exerts a significant effect on its function. Furthermore, the propensity of the 150-loop in 09N1 towards the closed state may be a major factor in determining the advantage over the $18 \mathrm{~N} 1$ in regards to pairing with $18 \mathrm{H} 1$. One might speculate that if the 09N1 has higher activity than $18 \mathrm{~N} 1$ than it is better balanced with $18 \mathrm{H} 1$ which may have better entry ability than $09 \mathrm{H} 1$ (or the opposite situation), however further experiments are necessary to arrive at any conclusion.

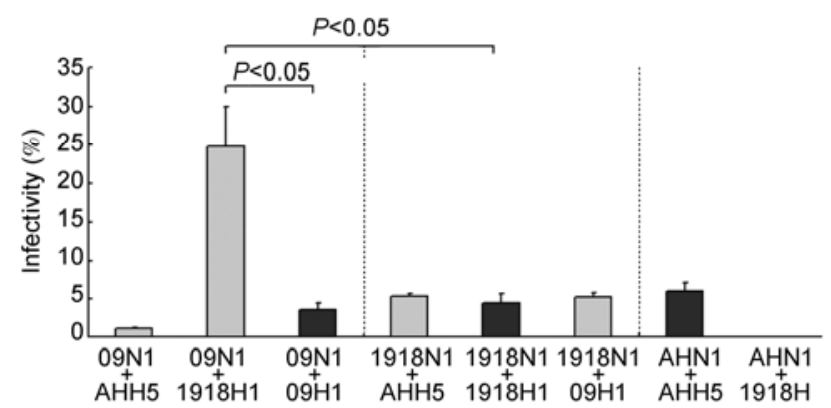

Figure 4 Infectivity of normalized influenza pseudotyped particles (pps) with various HA/NA combinations; infectivity is presented as the Mean+/SD percentage of infected cells from 3 repeats. All pps were grouped by NA; 2009 H1N1, 1918 H1N1, and H5N1 (A/Anhui/1/2005) are abbreviated as 09, 1918, and $\mathrm{AH}$, respectively. Reprinted from Zhang et al. [46].

There has been a great deal of interest and research into the $\alpha 2,3$ and $\alpha 2,6$ specificity of HA and the structural basis of substrate binding. Some studies have investigated the specificity of various NAs to $\alpha 2,3$ vs $\alpha 2,6$ linked receptor substrates $[47,48]$, however no specificity rule for NA has been firmly established and the structural basis of the binding to $\alpha 2,3$ or $\alpha 2,6$ substrates is uncharacterized. The detailed characterization of NA specificity is essential to understanding HA-NA pairing. Further research regarding the receptor recognition of both $\mathrm{HA}$ and NA including not only specificity but relative affinity and covering a variety of prevalent mammalian sialic acid containing receptors will provide a strong basis for the mechanisms of HA and NA pairing. This will contribute to the comprehensive understanding of influenza virus re-assortment.

The authors thank Dr. Yoshihiro Kawaoka and Dr. Takeshi Noda for providing the electron micrograph image of influenza virions for our cover design. This work was supported by Chinese Academy of Sciences Research Fellowship for Young International Scientists (2010Y2SB12) and the National Natural Science Foundation of China for International Young
Scientists (31050110126) to Vavricka CJ, and the National Natural Science Foundation of China (81021003) to Gao GF.

1 Potter C W. Influenza. Amsterdam, Boston: Elsevier, 2002

2 Kumar A, Zarychanski R, Pinto R, et al. Critically ill patients with 2009 influenza A (H1N1) infection in Canada. JAMA, 2009, 302: 1872-1879

3 Wise H M, Foeglein A, Sun J, et al. A complicated message: Identification of a novel PB1-related protein translated from influenza A virus segment 2 mRNA. J Virol, 2009, 83: 8021-8031

4 Gamblin S J, Skehel J J. Influenza hemagglutinin and neuraminidase membrane glycoproteins. J Biol Chem, 2010, 285: 28403-28409

5 Liu D, Liu X, Yan J, et al. Interspecies transmission and host restriction of avian H5N1 influenza virus. Sci China Life Sci, 2009, 52: $428-438$

6 Zhang W, Qi J X, Shi Y, et al. Crystal structure of the swine-origin A (H1N1) - 2009 influenza A virus hemagglutinin (HA) reveals similar antigenicity to that of the 1918 pandemic virus. Protein Cell, 2010, 1: 459-467

7 Hay A J, Gregory V, Douglas A R, et al. The evolution of human influenza viruses. Philos T Roy Soc B, 2001, 356: 1861-1870

8 Smith G J, Vijaykrishna D, Bahl J, et al. Origins and evolutionary genomics of the 2009 swine-origin H1N1 influenza A epidemic. Nature, 2009, 459: 1122-1125

9 Gao G F, Sun Y. It is not just AIV: From avian to swine-origin influenza virus. Sci China Life Sci, 2010, 53: 151-153

10 Neumann G, Noda T, Kawaoka Y. Emergence and pandemic potential of swine-origin H1N1 influenza virus. Nature, 2009, 459: 931939

11 Kawaoka Y, Krauss S, Webster R G. Avian-to-human transmission of the $\mathrm{Pbl}$ gene of influenza-a viruses in the 1957 and 1968 Pandemics. J Virol, 1989, 63: 4603-4608

12 WHO. Evolution of H5N1 avian influenza viruses in Asia. Emerg Infect Dis, 2005, 11: 1515-1521

13 Li C J, Hatta M, Nidom C A, et al. Reassortment between avian H5N1 and human H3N2 influenza viruses creates hybrid viruses with substantial virulence. Proc Natl Acad Sci USA, 2010, 107: 46874692

14 Ma W, Lager K M, Vincent A L, et al. The role of swine in the generation of novel influenza viruses. Zoonoses Public Health, 2009, 56 : 326-337

15 Nicholls J M, Bourne A J, Chen H, et al. Sialic acid receptor detection in the human respiratory tract: Evidence for widespread distribution of potential binding sites for human and avian influenza viruses. Respir Res, 2007, 8: 73

16 Nelli R K, Kuchipudi S V, White G A, et al. Comparative distribution of human and avian type sialic acid influenza receptors in the pig. BMC Vet Res, 2010, 6: 4

17 Shinya K, Ebina M, Yamada S, et al. Avian flu: Influenza virus receptors in the human airway. Nature, 2006, 440: 435-436

18 Zhao G P, Zhong Y. Learn to fight from the war. Chinese Sci Bull, 2009, 54: 2157-2158

19 Kou Z, Hu S N, Li T X. Genome evolution of novel influenza A (H1N1) viruses in humans. Chinese Sci Bull, 2009, 54: 2159-2163

20 Chen J M, Sun Y X, Liu S, et al. Origin and future distribution of the new A (H1N1) influenza virus emerging in North America in 2009. Chinese Sci Bull, 2009, 54: 2174-2178

21 Wu B, Wang C M, Dong G Y, et al. Molecular characterization of $\mathrm{H} 1 \mathrm{~N} 1$ influenza A viruses from human cases in North America. Chinese Sci Bull, 2009, 54: 2179-2192

22 Skehel J J, Wiley D C. Receptor binding and membrane fusion in virus entry: The influenza hemagglutinin. Annu Rev Biochem, 2000, 69: 531-569

$23 \mathrm{Wu} \mathrm{D}, \mathrm{Xu} \mathrm{T} \mathrm{L}$, Sun J, et al. Structure modeling and spatial epitope analysis for HA protein of the novel H1N1 influenza virus. Chinese Sci Bull, 2009, 54: 2171-2173

24 Yan A, Ding G H, Zhou Z F, et al. Subtyping of type A influenza by sequencing the variable regions of HA gene specifically amplified 
with RT-PCR. Chinese Sci Bull, 2009, 54: 2164-2167

25 He Y G, Ding G H, Bian C, et al. The mutation network for the hemagglutinin gene from the novel influenza A (H1N1) virus. Chinese Sci Bull, 2009, 54: 2168-2170

26 Xu R, Ekiert D C, Krause J C, et al. Structural basis of preexisting immunity to the 2009 H1N1 pandemic influenza virus. Science, 2010, 328: 357-360

27 Yang H, Carney P, Stevens J. Structure and receptor binding properties of a pandemic H1N1 virus hemagglutinin. PLoS Curr Influenza, 2010, 2: RRN1152

28 Wrammert J, Koutsonanos D, Li G M, et al. Broadly cross-reactive antibodies dominate the human B cell response against 2009 pandemic H1N1 influenza virus infection. J Exp Med, 2011, 208: 181-193

29 Wang C C, Chen J R, Tseng Y C, et al. Glycans on influenza hemagglutinin affect receptor binding and immune response. Proc Natl Acad Sci USA, 2009, 106: 18137-18142

30 Matrosovich M N, Matrosovich T Y, Gray T, et al. Human and avian influenza viruses target different cell types in cultures of human airway epithelium. Proc Natl Acad Sci USA, 2004, 101: 4620-4624

31 Stevens J, Blixt O, Glaser L, et al. Glycan microarray analysis of the hemagglutinins from modern and pandemic influenza viruses reveals different receptor specificities. J Mol Biol, 2006, 355: 1143-1155

32 Gamblin S J, Haire L F, Russell R J, et al. The structure and receptor binding properties of the 1918 influenza hemagglutinin. Science, 2004, 303: 1838-1842

33 Reid A H, Janczewski T A, Lourens R M, et al. 1918 influenza pandemic caused by highly conserved viruses with two receptor-binding variants. Emerg Infect Dis, 2003, 9: 1249-1253

34 Tumpey T M, Maines T R, Van Hoeven N, et al. A two-amino acid change in the hemagglutinin of the 1918 influenza virus abolishes transmission. Science, 2007, 315: 655-659

35 Liu Y, Childs R A, Matrosovich T, et al. Altered receptor specificity and cell tropism of D222G hemagglutinin mutants isolated from fatal cases of pandemic A (H1N1) 2009 influenza virus. J Virol, 2010, 84: 12069-12074

36 Sun Y, Shi Y, Zhang W, et al. In silico characterization of the functional and structural modules of the hemagglutinin protein from the swine-origin influenza virus A (H1N1)-2009. Sci China Life Sci,
2010, 53: 633-642

37 Stevens J, Corper A L, Basler C F, et al. Structure of the uncleaved human $\mathrm{H} 1$ hemagglutinin from the extinct 1918 influenza virus. Science, 2004, 303: 1866-1870

38 Russell R J, Haire L F, Stevens D J, et al. The structure of H5N1 avian influenza neuraminidase suggests new opportunities for drug design. Nature, 2006, 443: 45-49

39 Li Q, Qi J, Zhang W, et al. The 2009 pandemic H1N1 neuraminidase N1 lacks the 150-cavity in its active site. Nat Struct Mol Biol, 2010, 17: $1266-1268$

40 Amaro R E, Minh D D, Cheng L S, et al. Remarkable loop flexibility in avian influenza N1 and its implications for antiviral drug design. J Am Chem Soc, 2007, 129: 7764-7765

41 von Itzstein M. The war against influenza: Discovery and development of sialidase inhibitors. Nat Rev Drug Discov, 2007, 6: 967-974

42 Varghese J N, Smith P W, Sollis S L, et al. Drug design against a shifting target: A structural basis for resistance to inhibitors in a variant of influenza virus neuraminidase. Structure, 1998, 6: 735-746

43 Amaya M F, Watts A G, Damager I, et al. Structural insights into the catalytic mechanism of Trypanosoma cruzi trans-sialidase. Structure, 2004, 12: 775-784

44 Watts A G, Oppezzo P, Withers S G, et al. Structural and kinetic analysis of two covalent sialosyl-enzyme intermediates on Trypanosoma rangeli sialidase. J Biol Chem, 2006, 281: 4149-4155

45 Watts A G, Damager I, Amaya M L, et al. Trypanosoma cruzi transsialidase operates through a covalent sialyl-enzyme intermediate: Tyrosine is the catalytic nucleophile. J Am Chem Soc, 2003, 125: 7532-7533

46 Zhang Y, Lin X, Wang G, et al. Neuraminidase and hemagglutinin matching patterns of a highly pathogenic avian and two pandemic H1N1 influenza A viruses. PLoS ONE, 2010, 5: e9167

47 Katinger D, Mochalova L, Chinarev A, et al. Specificity of neuraminidase activity from influenza viruses isolated in different hosts tested with novel substrates. Arch Virol, 2004, 149: 2131-2140

48 Xu G, Suzuki T, Maejima Y, et al. Sialidase of swine influenza A viruses: Variation of the recognition specificities for sialyl linkages and for the molecular species of sialic acid with the year of isolation. Glycoconj J, 1995, 12: 156-161

Open Access This article is distributed under the terms of the Creative Commons Attribution License which permits any use, distribution, and reproduction in any medium, provided the original author(s) and source are credited. 